\title{
Empoderamiento femenino, una perspectiva de tres generaciones en mujeres urbanas de Mérida, Yucatán, México
}

\section{Women empowerment, a three-generation perspective on urban women from Mérida, Yucatán, México}

DOI: http://dx.doi.org/10.17981/cultedusoc.10.1.2019.11

Recibido: 29/05/2018 Aceptado: 28/10/2018

Laura Isabel Alamilla Herrera

Universidad Autónoma de Yucatán (México) laualamilla@gmail.com

Andrea Itzel Trucios Lara

Universidad Autónoma de Yucatán (México) itztrucios@gmail.com

Para citar este artículo:

Alamilla, L. y Trucios, A. (2019). Empoderamiento femenino, una perspectiva de tres generaciones en mujeres urbanas de Mérida, Yucatán, México. Cultura, Educación y Sociedad, 10(1). 167-179. DOI: http://dx.doi.org/10.17981/cultedusoc.10.1.2019.11

\section{Resumen}

La evolución del empoderamiento femenino a través del tiempo se considera un tema necesario para observar los cambios y transformaciones ocurridas en las diferentes generaciones. El presente artículo pretende analizar los niveles de empoderamiento de mujeres urbanas de Mérida, (Yucatán, México), e identificar las principales variables que afectan el referido proceso. La metodología está basada en la aplicación de una escala y de dos premisas, en una muestra conformada por 191 mujeres de un rango de edad entre 18 a 73 años, las cuales se clasifican en tres generaciones. Los resultados evidenciaron la inexistencia de diferencias en los niveles de empoderamiento femenino entre las generaciones, en cambio, se encontraron correlaciones en dos de las siete dimensiones del empoderamiento. Se avala la tesis de que la contemporaneidad es el factor más influyente en los grados y formas de empoderamiento. Se concluye por lo tanto que, aunque no se tengan las herramientas para empoderarse, existe una conciencia de la necesidad de un cambio orientador de las múltiples posibilidades del empoderamiento femenino a partir de sus propias potencialidades.

Palabras clave: Empoderamiento femenino; persepectiva generacional; mujer urbana.

\begin{abstract}
The evolution of female empowerment through time is considered a necessary theme to observe the changes and transformations that have occurred in the different generations. This article seeks to analyze the levels of empowerment of urban women in Merida (Yucatan, Mexico), and identify the main variables that affect the process. The methodology is based on the application of a scale and two premises, in a sample made up of 191 women between the ages of 18 and 73, who are classified into three generations. The results showed that there were no differences in the levels of female empowerment between the generations, whereas correlations were found in two of the seven dimensions of empowerment. The thesis is supported that contemporaneity is the most influential factor in the degrees and forms of empowerment. It is therefore concluded that, even if one does not have the tools to empower oneself, there is an awareness of the need for a guiding change in the multiple possibilities of female empowerment based on one's own potentialities.
\end{abstract}

Keywords: Feminine empowerment; persepective generational; urban woman. 


\section{INTRODUCCIÓN}

La principal problemática de esta investigación recae sobre el Empoderamiento Femenino, definido por Schuler (1997) como "un proceso por medio del cual las mujeres incrementan la capacidad de configurar sus propias vidas y su entorno; una evolución en la concientización de las mujeres sobre sí mismas, en su estatus y en su eficacia en las interacciones sociales". Este empoderamiento es necesario para lograr una mejor independencia, tenacidad y seguridad propia necesarios para el desarrollo de la mujer mexicana y la construcción de su realidad a partir de normas culturales que se basan en el predominio de los hombres sobre las mujeres (Díaz, 1979); dicho lo anterior, se debe mencionar que el Empoderamiento Femenino se logra en correspondencia con el cumplimiento de la igualdad de género. Emakunde (2013) declara la ausencia de información de carácter cuantitativo, así como también en cuanto al empoderamiento con las generaciones, en ambos casos, en México son pocas las investigaciones; de allí la importancia y pertinencia de esta investigación donde se consideran los factores que empoderan a las mujeres de acuerdo a cada generación.

La evolución del empoderamiento femenino a través de las generaciones se considera un tema necesario para observar el cambio y comparar los niveles entre sí, identificando si realmente el contexto histórico y social actual participa en el proceso de cambio individual y así tener un conocimiento más completo al identificar en qué dimensiones del empoderamiento se tienen niveles más bajos, y según la generación estudiada poder construir la estrategia más adecuada e impulsar procesos de empoderamiento del género femenino. Autores como Robles (2015), destacan la im- portancia del estudio del género como base para el fortalecimiento de los derechos humanos que coadyuvan a la formación integral del individuo; de allí se infiere la necesidad de generar espacios socialmente responsables (Crasto, Marín-González y Senior, 2016) donde tanto individuos como organizaciones desarrollen sus potencialidades y puedan empoderarse de acuerdo a su perfil de capacidades.

Consecuentemente, el artículo se orienta a describir el nivel de empoderamiento presente en las mujeres urbanas en la ciudad de Mérida, (Yucatán, México), con énfasis en las diferencias entre una generación y otra, lo que conlleva a establecer un sentido de correspondencia entre las dimensiones del empoderamiento femenino y la generación a la que pertenecen.

\section{Antecedentes}

\section{Generaciones}

Una generación es un conjunto de personas que tienen características distintivas, han crecido y vivido experiencias formativas vinculadas con el tiempo y el lugar (Ogg $\&$ Bonvalet, 2006; Ferreiro, 2006). No hay un rango de edades y de nomenclatura estandarizados, existen diferencias en las definiciones de cada autor, sin embargo, para efectos de esta investigación, las generaciones consideradas son las siguientes: millennials, generación X y baby boomers. Se denominaron millennials a las mujeres que han nacido entre 1999 y 1986 (Smola y Sutton, 2002; Duchscher y Cowin, 2004; Chirinos, 2009; Furlow, 2011), si han nacido entre 1985 y 1965 se dirá que pertenecen a la generación $X$ (Duchscher y Cowin, 2004; Chirinos, 2009) y se les llamará baby boomers si nacieron entre 1964 y 1944 (Duchscher y Corwin, 2004; Ferreiro, 2006; Chirinos, 2009). 
Generación Baby boomers

Los baby boomers son la generación más antigua. Chirinos (2009) los describe como idealistas con deseos de transformar el mundo y no se complacen solo con lo que les fue dado; son introvertidos, moralistas, pretenciosos y seguros de sí mismos. En el contexto mexicano, como factor de influencia de esta generación, destaca que entre 1940 y 1950 la cobertura educativa continuó creciendo, no obstante, el acceso al sistema educativo seguía siendo complejo. Es una generación que vivió con un sólo canal de televisión y probablemente una o dos marcas de refrescos y un estreno cinematográfico semanal (Ferreiro, 2006) y los procesos como la globalización no habían sido consolidados a nivel mundial. Estudios empíricos afirman el estereotipo de trabajadores de los baby boomers, siendo elementos indispensables para los compañeros de trabajo de diferentes generaciones aunque estos no compartan los mismos valores (McGuire, Todnem By y Hutchings, 2007).

\section{Generación X}

Las personas de la generación $X$ han sido criadas de manera independiente y han presenciado numerosos divorcios (Chirinos, 2009). Según Martin (2005), en el ámbito laboral los pertenecientes a esta generación les gusta trabajar de manera autónoma y notoria y manifiestan desacuerdos con reuniones y grupos de trabajo. Para la década de los ochenta, se comienza a trabajar con las mujeres, proporcionando asesoría en foros internacionales y desarrollando acciones que permiten el avance e inserción en el ámbito laboral; esto representa un elemento importante para el proceso de inclusión y empoderamiento, que ha implicado cambios en la ideología prevaleciente en la actualidad (Camberos, 2011). Las mujeres de esta generación al empezar a tener ingresos económicos propios aumentan su poder de negociación a nivel familiar y su capacidad de supervivencia fuera de ésta (Agarwal, 1999, citado en Pérez y Vázquez, 2008). La contribución en la economía familiar repercute positivamente en el reconocimiento social y el empoderamiento (Mayoux, 1997, citado en Pérez y Vázquez, 2008), por lo tanto, al adquirir una independencia económica se promueve su empoderamiento (Hernández y García, 2015).

\section{Generación Millenial}

La generación millennial reconocida por nacer en tiempos más prósperos (Pincheira y Arenas, 2016) y en la era de la tecnología (Ferreiro, 2006) ha evidenciado una fácil y rápida adaptación, además de mostrar tendencias vanguardistas e innovadoras (Chirinos, 2009). La disposición de tecnología permite el acceso al conocimiento a nivel mundial, lo cual es otra característica de esta generación: Nacer en la era de la globalización. Los millennials tienen un mayor acceso a la educación a comparación a las generaciones anteriores. En Beijing, se celebró la IV Conferencia Mundial sobre mujeres (1995), en la cual, adquiere particular relevancia el tema del empoderamiento como estrategia para el cambio (Pérez y Vásquez, 2009). Esta generación crece en una época donde se debaten temas como el empoderamiento y se empiezan a impulsar las políticas públicas con perspectiva de género, las cuales garantizan una sociedad ecuánime en derechos y oportunidades para mujeres y hombres y no sociedades creadas en favor de un sexo en particular (Hernández y García, 2011). 


\section{Un poco de historia}

En 1923 Yucatán es el primer estado que durante la gubernatura de Felipe Carrillo Puerto las mujeres ocuparon puestos de representación popular, siendo Elvia Carrillo Puerto, Raquel Dzib y Beatriz Peniche electas para diputadas al Congreso Estatal y Rosa Torre electa para regidora en el ayuntamiento de Mérida, no obstante, dejarían sus puestos tras el asesinato del gobernador. Para el año 1953, específicamente, el 17 de octubre es reconocido el derecho de la mujer a votar, así como también para ser elegida. Aunque, el tener el derecho al voto no es suficiente, Sánchez (2006) afirma la importancia de transformar la imagen de las mujeres, observándolas como sujetos protagónicos en la toma de decisiones, esto supone encontrar las vías necesarias a recorrer para la construcción de su ciudadanía plena, la cual pretende: superar su exclusión por largo tiempo, incluir la diversidad, influir como grupo en todos los espacios de decisión, así como generar condiciones, recursos, poderes y acciones afirmativas para lograr el empoderamiento.

Una de las vías para construir esta ciudadanía es la necesidad de fomentar la participación de las mujeres en la sociedad, sobre todo en los ámbitos de toma de decisiones, como la política, y para esto se requiere construir nuevas identidades femeninas, fortalecidas y no denigradas por una cultura que considera con mayor valor al sexo masculino; y por consiguiente crear las bases para un fortalecimiento y empoderamiento de la mujer.

En México la inequidad de género es evidente, sobre todo en el ejercicio de los derechos políticos y civiles de las mujeres; destacando en aspectos como el derecho a postularse para cargos públicos, los cuales son ejercidos mayormente por los varones. "Para las mujeres es un desafío la ideología patriarcal, con miras a transformar las estructuras que refuerzan la discriminación de género y la desigualdad social" (Camberos, 2011). El estereotipo de "macho" y la cultura machista en México, son factores que han propiciado la sumisión en las mujeres, y a su vez, es un factor detonante para el proceso de empoderamiento, es decir, la persona debe haber estado desempoderada para que el empoderamiento se presente (Hernández y García, 2015).

\section{Empoderamiento}

El empoderamiento no es un suceso estático, sino al contrario, es un proceso gradual y dinámico de los individuos en el cual la autonomía y la toma de decisiones son indispensables para dicho proceso; llega a influenciar en los diversos contextos donde se desenvuelve y su impacto ocupa dos niveles: Individual y colectivo o social (Hernández y García, 2015). El primero, es el empoderamiento individual (intrínseco) entendido como "la capacidad individual para modificar el contexto con base en una motivación intrínseca de cambio" (Pick \& Sirkin, 2010. Autores como Pick, Sirkin, Ortega, Osorio y Martínez (2007) hablan de el empoderamiento como parte de la agencia personal la cual "se refiere a la competencia personal, enfocándose principalmente en el individuo como un actor que opera en un contexto social" por lo tanto, la agencia personal tiene consecuencias que repercuten a nivel social y cuando impacta a la familia, colegas, organizaciones y comunidad, se convierte en empoderamiento, está vinculada con conceptos como la autoestima, autonomía, control, autorregulación, autoeficacia, los cuales coinciden con la teoría del empoderamiento. 
Diversos autores confirman como la autonomía es fundamental para el proceso de empoderamiento (Casique-Rodríguez, 2014; Emakunde, 2013; Vidal, 2015). Cañedo-Cázarez y Mendoza-Guerrero (2017) encuentran la participación en el ámbito público y toma de decisiones influyente en el proceso de empoderamiento y también el poder participar en la resolución de conflictos y negociar con los representantes del poder formal. El poder para el desarrollo de nuevas habilidades como la gestión, la creación de estrategias, la resistencia civil, el poder para mostrar la capacidad de organización y la concreción de alianzas y el poder para aumentar la confianza en sí mismo, así como también alcanzar objetivos no sólo con la vida privada sino con la colectividad (Cañedo y Mendoza, 2017).

Un referente importante según Erazo, Jiménez y Lopéz (2014) plantea la importancia del papel de las mujeres en la construcción de espacios públicos y su incidencia en la ejecución de un proyecto participativo. De igual forma, en el país vasco, las mujeres que participaron en movimientos sociales de mujeres muestran indicadores más altos en cuanto a los progresos en las condiciones de igualdad de las mujeres (Emakunde, 2013), siendo así, la participación social factor que influye en las dimensiones del empoderamiento.

Emakunde (2013) en su estudio, descubre cinco indicadores que fortalecen el empoderamiento: el primer indicador es una autonomía a nivel individual, sin embargo, en el ámbito laboral se tiene un nivel inferior en avances, con obstáculos significativos; el segundo indicador se refiere a que las mujeres perciben un avance en el factor de igualdad de oportunidades al comparar con generaciones anteriores; el tercer factor demuestra que las mujeres de 18 a 64 años consideran que existen mayores oportunidades (o menores limitaciones) para que las mujeres estén presentes en ámbitos de carácter social.

Un cuarto indicador, expone el incremento en la accesibilidad formal en el ámbito social es un reflejo de la búsqueda de mitigar las situaciones de discriminación, no obstante, se siguen dando, en especial en el ámbito laboral y por la prevalencia del machismo como cultura; el quinto indicador, el valor de la autonomía individual se canaliza fundamentalmente en el plano personal -en la capacidad para tener o hacer nuevas amistades/relaciones, en la expresión de sus propias opiniones, en las relaciones de pareja, en la gestión del dinero, en el cuidado de otras personas, o en la elección de lo que quiere hacer o gestión de su tiempo, si bien en este último plano se identifican mayores limitaciones por parte de aquéllas que experimentan la denominada "dualidad de tareas"-, donde las mujeres consideran que ya vienen tomando, mayoritariamente, sus decisiones en forma autónoma.

En el estudio pionero de Escudero, Serrano-Barquín, Segrado, Serrano y López (2017) en el municipio de Metepec (México), las mujeres que laboran en establecimientos de hospedaje a pesar a desempeñar funciones de rango bajo, se perciben niveles de empoderamiento altos, debido a factores como el recibir un salario propio, ayudar en los gastos familiares, salir de sus hogares, sentir respeto y ser valoradas fuera del ámbito hogareño, afirmando el empoderamiento de la mujer en Metepec y su relación con el desarrollo de sus cualidades personales.

Según Pick, García y Leenen (2011), las mujeres con un nivel educativo de media superior o mayor, tienen un mejor desempeño al momento de resolver conflictos y una mayor disposición a abandonar actitudes y conductas que les impidan cambiar su entorno, siento este último el empoderamiento negativo, que las mujeres de una menor escolaridad. 
En conclusión, el empoderamiento abarca desde un nivel individual y social, implica procesos cognitivos, psicológicos, políticos y económicos (Hernández y García, 2015) y es fuertemente influenciado por la cultura e historia del país, en este caso, México. Se evidencian constantes cambios con importantes hitos que han marcado la evolución y desarrollo de las distintas generaciones en cuanto al tema tratado. Una constante historica en el machismo ha sido la limitación de las mujeres para realizar actividades de carácter privado, en su mayoría, son infravaloradas por la sociedad. De por si, la cultura mexicana es en esencia machista, lo cual ocasiona distorsiones que afectan la relación de hombres y mujeres en todos los ámbitos: emocional, sexual, laboral, político (Castañeda, 2007), no obstante, esto es una causa para que se dé el proceso de empoderamiento.

Factores como la autonomía, la representación y participación política influyen positivamente en el proceso de empoderamiento femenino, pero este proceso es multidimensional por tanto un proceso de empoderamiento de las mujeres, debe gestionarse desde diversos campos, niveles y disciplinas (Hernández y García, 2015).

\section{Metodología}

\section{Diseño de investigación}

Este artículo se aborda desde un enfoque cuantitativo, con alcance confirmatorio y de tipo ex post facto. Las variables abordadas son:

Empoderamiento, de acuerdo con Schuler (1997) es "un proceso por medio del cual las mujeres incrementan su capacidad de configurar sus propias vidas y su entorno; una evolución en la concientización de las mujeres sobre sí mismas, en su estatus y en su eficacia en las interacciones sociales."
Generación, según Ogg y Bonvalet (2006) se define como la idea de una experiencia formativa de actitudes y conductas, y es llevada colectivamente por los individuos durante el curso de su vida.

La población considerada para esta investigación son mujeres urbanas de Mérida Yucatán, divididas en tres generaciones baby boomers nacidos de 1944 a 1964 (53 a 73 años de edad), generación $x$ nacidos de 1985 a 1965 (32 a 52 años de edad) y millennials nacidos de 1986 a 1999 (18 a 31 años de edad). La muestra consta de 70 participantes para la generación millennials, 60 participantes para la generación $x$ y 70 para las baby boomers, sumando así un total de 200 mujeres elegidas de manera intencional. La recolección de estos datos se desarrolla a través de encuestas por medio de las redes sociales y debido a la falta de respuesta electrónica de 40 mujeres baby boomers, se les aplicó el instrumento de manera personal.

Se utilizó el Instrumento para la $\mathrm{Me}$ dición de Empoderamiento en Mujeres (IMEM) de Hernández y García (2008) con validez para su aplicación a nivel nacional en mujeres de un rango de edades de 15 a 73 años. Este instrumento abarca las siguientes dimensiones del empoderamiento: empoderamiento participativo, temeridad, influencias externas, independencia, igualdad, satisfacción social y seguridad, calificados de manera individual teniendo cada una de 4 a 7 reactivos por dimensión. El instrumento clasifica las respuestas en tres puntajes siendo: alto (mayor a 105 puntos) medio, (de 90 a 104 puntos) y bajo (menor a 89 puntos).

Se utilizó un instrumento complementario que constó de dos premisas para conocer las experiencias de las participantes, La preguntas son: "Comparta una experiencia en donde haya vivido una situación de empoderamiento" y "comparta una 
experiencia en donde haya sentido que no pudo empoderarse"

La sistematización procedimental implicó solicitar autorización para la difusión del IMEM a sus autores. Posteriormente, se adaptó la escala a formato digitalizado con las premisas en la aplicación de Google Forms; se promocionó en una página en la red social Facebook.

Al no lograr el objetivo de 200 participantes, debido a que no se alcanzó el mínimo de mujeres pertenecientes a la generación baby boomer, se acudió a diversos lugares públicos en donde a 40 mujeres de la generación baby boomer se les aplicó la escala, este tuvo un formato similar al digital, explicándoles el propósito de la investigación a las participantes, dejando en claro que su participación es completamente voluntaria y confidencial, se les entregó el instrumento a las que hayan accedido a responder el mismo explicándoles las instrucciones. En forma simultánea se recabaron por internet los datos de las encuestas. Los resultados se procesaron a través del software de análisis de datos cuantitativos Statistical Package for Social Science (SPSS).

\section{Resultados}

Se realizan los estudios descriptivos para la muestra total. El IMEM clasifica el empoderamiento en tres puntajes, siendo alto (mayor a 105 puntos) medio (de 90 a 104 puntos) y bajo (menor a 89 puntos). El empoderamiento en general de las mujeres urbanas de Mérida, Yucatán, tuvo una media $\mathrm{M}=103.25$ puntos, alcanzando así, un nivel de empoderamiento medio cercano al alto.

Para comprobar cuáles son los niveles de empoderamiento que presenta cada generación se compararon las medias de los puntajes de cada una, obteniendo así, generación millenial $\mathrm{M}=102.71$ puntos, generación $x$ con $\mathrm{M}=104.22$ puntos y generación baby boomer con $\mathrm{M}=102.71$ puntos, todas con un nivel de empoderamiento medio cercano al alto, siendo la generación $\mathrm{x}$ la que obtuvo el puntaje de empoderamiento más alto de las tres generaciones. Estos resultados rechazan la hipótesis de que la generación millennials presenta niveles más altos de empoderamiento femenino debido a que las tres generaciones presentan una media similar en los puntajes de empoderamiento.

Se encontró $\square$ una correlación en los puntajes de las dimensiones de temeridad e influencias externas, los cuales, disminuyen a medida que la edad de las mujeres participantes aumenta.

TABLA 1.

Correlación de significancia:

años cumplidos y dimensiones

Correlación de significancia: años cumplidos y dimensiones

\begin{tabular}{lll}
\hline Temeridad & Pearson Correlation & -.154 \\
& Sig. (2-tailed) & .037 \\
& $\mathrm{~N}$ & 184 \\
$\begin{array}{l}\text { Influencias } \\
\text { externas }\end{array}$ & Pearson Correlation & .172 \\
& & \\
& Sig. (2-tailed) & .020 \\
& $\mathrm{~N}$ & 183 \\
\hline
\end{tabular}

Después de responder al instrumento, las mujeres desarrollaron dos enunciados en los que contaban experiencias de empoderamiento y no empoderamiento; la intención encontrar patrones o rasgos comunes en las respuestas, así como también en relación al contexto en los que suceden estas situaciones como son el familiar, escolar, laboral, entre otros. 
TABLA 2

Incidencia por generación: empoderamiento

\begin{tabular}{lcccl}
\hline \multicolumn{5}{c}{ Incidencia por generación: empoderamiento } \\
\hline Constructo & Millenial & Generación x & Baby boomer & Total \\
Logro & 16 & 21 & 16 & 53 \\
Libertad & 31 & 05 & 12 & 48 \\
Decisión & 03 & 17 & 14 & 34 \\
Autonomía & 21 & 03 & 0 & 24 \\
Independencia & 07 & 11 & 03 & 21 \\
Liderazgo & 09 & 02 & 03 & 14 \\
Oportunidades & 01 & 0 & 0 & 01 \\
Altruismo & 0 & 1 & 0 & 01 \\
Experiencia & 0 & 0 & 01 & 01 \\
\hline
\end{tabular}

Hubo un total de 9 constructos que surgieron después de un extenso análisis de las respuestas de las mujeres en cuanto al enunciado: "Comparta una experiencia en la que se haya sentido empoderada". La incidencia de estos constructos por generación se puede visualizar en la anterior Tabla 2.

En el plano académico el mayor logro es cuando se sienten satisfechas consigo mismas o incluso reciben algún reconocimiento; en este caso las millennials resaltan el hecho de titularse tanto de sus estudios de pregrado como de la maestría; también se empoderan al ser elegidas representantes de grupo o al momento de sus procesos formativos cuando exponen temáticas frente a sus compañeros.

En el contexto laboral, las millennials expresaron su empoderamiento en situaciones donde se les solicitaba su apoyo $u$ obtenían un puesto debido a sus conocimientos o cuando las promocionaban en sus cargos; las mujeres que pertenecen a la generación $x$ fueron las que en mayor grado destacaron situaciones de logro, siendo un total de 19 incidencias, en donde el contexto laboral fue el más mencionado. Hablaron de empoderarse a través de la creación de su propio negocio, del ascenso laboral en cargos donde tradicionalmente se ubican hombres, por ejemplo ser gerentes en la empresa en donde trabajan.

En el contexto económico, el proceso de empoderamiento se da con la obtención de bienes materiales y la posibilidad de apoyar a su familia en una adversidad; Para las baby boomers, el logro se dio en el contexto familiar cuando apoyan a sus hijos en los estudios universitarios; en el contexto laboral, el fundar una sociedad civil, obtener el puesto que se desea y lograr un ascenso representan situaciones de empoderamiento para las mujeres.

Las baby boomers también hablan del logro en la parte económica, en donde la obtención de ingresos propios y aumentos de sueldo fueron temas mencionados; en general las mujeres hablaron de situaciones en donde alcanzan una meta o destacan en el ámbito en el que se desempeñan, logrando así, un empoderamiento. 
El constructo con más incidencia en la generación millennials fue la libertad; en el contexto escolar las tres generaciones mencionaron que su empoderamiento se dio al momento de elegir libremente su carrera universitaria; en el contexto laboral, fue la libre elección de su lugar de trabajo, igualmente, las millennials destacaron la libertad de expresión, en donde se sintieron empoderadas al poder opinar y manifestar su ideología; también hubo otros casos de libertad como es la libertad personal y corporal, donde las mujeres se empoderan al expresar su sexualidad libremente y elegir la ropa que quieren usar.

Para el constructo "decisión", las millennials casi no tocaron el tema, más que empoderarse al sentir que toman las decisiones sobre su propia vida; en el caso de la generación $X$ y las baby boomers, los temas fueron muy similares, el hecho de planificar su familia, la decisión de tener o no hijos y con quién tenerlos representa una situación de empoderamiento; por su parte en el contexto laboral, las mujeres tomaron decisiones en relación a dejar de trabajar para dedicarse a su familia o por el contrario, continuar trabajando, las baby boomers mencionaron como situaciones de empoderamiento la toma de decisiones en relación a un viaje, ya sea para cuestiones escolares o simplemente conocer nuevas tierras.

El constructo autonomía, fue el segundo con más incidencia en la generación millennials; la mayoría de las veces en que las mujeres se sintieron empoderadas está relacionado con su elección de carrera, a pesar de opiniones contradictorias tanto de su familia como de las demás personas; en el ámbito laboral, consideran una experiencia de empode- ramiento emprender un nuevo negocio; en cuestiones personales, las mujeres eligen si tener o no hijos, al mantenerse firmes en esta decisión, lograron un empoderamiento; en la generación $X$, muy poco se habló de autonomía pero sí se abordó en cuanto a la elección de trabajo, las mujeres se sintieron empoderadas al momento de decidir qué es lo mejor paras ellas en su futuro profesional a pesar de los obstáculos que las demás personas intenten imponer; el tema autonomía para la generación baby boomer no fue tocado en ninguno de los casos.

La independencia fue muy popular para la generación $X$ pero las mujeres hablaban de temas muy diversos, desde la decisión de mudarse solas, hasta el sentir general de que son dueñas de su vida y toman solas sus propias decisiones con respecto a su salud y estudios, entre otras temáticas; en el caso de las millennials todas las mujeres hablan sobre dos temas principales, mudarse a otra residencia o independizarse; comenzar a trabajar y no depender económicamente de su familia, aunque no se muden; En las baby boomer solo hay dos casos y ambas hablan de cuestiones más económicas, como el hecho de decidir sobre sus finanzas.

Para el constructo liderazgo, las tres generaciones hacen énfasis al ámbito laboral, en donde se sienten empoderadas teniendo subordinados y teniendo también la capacidad de dirigir al personal o en sus proyectos en general.

Para el enunciado: "Comparta una experiencia en la que se haya sentido empoderada"; se encontraron 17 constructos, la incidencia de estos por generación se puede visualizar a continuación (véase Tabla 3). 
TABLA 3.

Incidencia por generación: no empoderamiento

\begin{tabular}{lcccc}
\hline \multicolumn{1}{c}{ Constructo } & Millenial & Generación x & Baby boomer & Total \\
\hline Impotencia & 24 & 20 & 20 & 64 \\
Sexismo & 12 & 5 & 2 & 19 \\
Acoso callejero & 12 & 0 & 0 & 12 \\
No ser tomada en cuenta & 5 & 4 & 0 & 9 \\
Prohibición & 6 & 0 & 0 & 6 \\
Vulnerabilidad & 6 & 0 & 0 & 6 \\
Sumisión & 3 & 1 & 2 & 6 \\
Represión & 4 & 1 & 0 & 5 \\
Dependencia & 2 & 1 & 1 & 4 \\
Limitación & 2 & 1 & 1 & 4 \\
Violencia & 2 & 1 & 0 & 3 \\
Relaciones interpersonales & 0 & 1 & 2 & 3 \\
Sexualización & 1 & 0 & 0 & 1 \\
Acoso sexual & 1 & 0 & 0 & 1 \\
Manipulación & 1 & 0 & 0 & 1 \\
Indecisión & 0 & 1 & 0 & 1 \\
Estereotipos de género & 0 & 1 & 0 & 1 \\
\hline
\end{tabular}

La impotencia se define como la falta de fuerza, poder o competencia para realizar una cosa, hacer que suceda u oponerle resistencia; Para las tres generaciones, el constructo impotencia fue ampliamente destacado, siendo 24,20 y 20 casos respectivamente; en el contexto laboral, se tocaron dos puntos importantes en las tres generaciones: sentirse incapaces de dirigir un cargo o tomar las decisiones que les corresponden y el hecho de que los hombres reciben más oportunidades y obtengan mejor salario u obtengan puestos que, a su sentir, ellas tienen mejores competencias para ello.

En el ámbito escolar, para la generación $X$ y las baby boomers, la razón de su impotencia era el hecho de no poder concluir sus estudios, ya sea porque dejaron la carrera por elección propia o por situaciones econó- micas, en las milennials solo hubo dos casos del ámbito escolar, en los que se menciona el haber perdido las elecciones para ser jefe de grupo y el no poder ir a estudiar al extranjero por cuestiones personales; también mencionaron situaciones en donde la manera de vestir afecta su empoderamiento, pues se sienten tanto inseguras como criticadas por la sociedad en general, de manera más amplia, las mujeres no se sienten empoderadas cuando no son capaces de tomar sus propias decisiones o cuando dependen de otras personas para lograr sus metas u objetivos.

Se identificaron diversas situaciones de sexismo que mencionan en su mayoría la generación millennials, es reiterativa la frase "por ser mujer" acompañada de estereotipos de género; en las tres generaciones 
se enfatiza que los hombres quieren hacer las cosas por ellas porque "ellas no pueden, no saben o son muy débiles" no se sienten empoderadas, el contexto que más se tocó en las tres generaciones fue el laboral, en donde los hombres suelen obtener mejores puestos de trabajo, les asignan tareas más "complejas" o ganan más sueldo por el simple hecho de ser hombres.

El acoso callejero es un tema mencionado exclusivamente por la generación millennials, en donde las mujeres no se sienten empoderadas al recibir piropos, miradas y gritos de hombres pues expresan no saber qué hacer en esos casos y simplemente lo dejan pasar. El constructo vulnerabilidad deriva precisamente del acoso callejero, de igual manera mencionado únicamente por millennials, en donde al recibir este acoso, tienen el deseo de defenderse pero al sentirse inseguras y desprotegidas no encuentran el valor para hacerlo.

El tema prohibición también fue tocado exclusivamente por las mujeres millennials, en donde no se sienten empoderadas cuando los padres o pareja no les dejan realizar algunas actividades como ir a conciertos o salir con amigos.

\section{Conclusiones}

Actualmente las tres generaciones de mujeres urbanas de la ciudad de Mérida (Yucatán, México) tienen un nivel similar de empoderamiento por la contemporaneidad de la sociedad y no por su papel al momento de su nacimiento.

Existe una correlación en los puntajes de las dimensiones de temeridad e influencias externas. Una temeridad baja significa una persona con temor, insegura en sus decisiones, con miedo de sus propias acciones, y un puntaje bajo en influencias externas indica una persona que considera que el liderazgo está en función de situaciones ajenas a ella.
Esto supone también una baja autonomía, en donde las personas sienten la presión de demandas, estándares, reglas y expectativas externas, además de sentir que están a la merced de dichas expectativas.

La autonomía es muy difícil de alcanzar para personas que están acostumbradas al control externo y dependen del mismo para su autoestima (Pick, Sirkin, Ortega, Osorio y Martínez, 2007). Estos bajos puntajes en las dimensiones, se pueden atribuir a cuestiones de cultura o educación familiar en donde el machismo y la represión de la mujer estan más arraigados en la sociedad conservadora yucateca en la que crecieron las baby boomers, el tema de la represión, sigue vigente pero ha disminuido considerablemente con el tiempo y con la lucha de la mujer, en la cual, las millennials tienen mayor oportunidad de toma de decisiones, de liderar y encontrar puestos de poder.

Las respuestas de las participantes al enunciado "comparte una situación en donde te hayas sentido empoderada" se clasifican en dos tipos, el primero, son situaciones que las han colocado en una posición de sumisión, en las cuales narran cómo sobrellevaron dicha situación y alcanzaron el empoderamiento, lo cual confirma que "para que el proceso de empoderamiento se presente, la persona debe haber estado desempoderada, es decir, en una posición de sumisión (Hernández y García, 2015), el segundo tipo de respuesta hace referencia a lograr cumplir los ideales que las mujeres tienen sobre sí mismas e implícitamente en sus respuestas también se encuentran situaciones de reconocimiento social sobre sus propias capacidades, asemejándose al estudio de Escudero, Serrano-Barquín, Segrado, Serrano y López (2017) donde el empoderamiento de las mujeres de Metepec se relaciona al desarrollo de sus cualidades personales como son el sentir respeto y sentirse valoradas fuera del ámbito privado. 


\section{REFERENCIAS}

Agarwal, B. (1999). Negociación y relaciones de género: Dentro y fuera de la unidad doméstica. Historia Agraria, 17, SEHA.

Camberos, M. (2011). Empoderamiento femenino y políticas públicas, una perspectiva desde las representaciones sociales de género. Entramado 7(2). 40-53.

Cañedo-Cázarez, S. y Mendoza-Guerrero, J. (2017). Desplazamiento forzado y empoderamiento femenino: el caso de la presa picachos en el sur de Sinaloa, México. El Ágora USB, 17(2), 370-386. https://doi. org/10.21500/16578031.3279

Casique-Rodríguez, I. (2014). Empoderamiento de las jóvenes mexicanas y prevención de la violencia en el noviazgo. Papeles de Población, 20(82) 27-56. Recuperado de http://www.redalyc. org/articulo.oa?id=11232827003

Castañeda, M. (2007). El machismo invisible regresa. Ed. Punto de lectura comunidades rurales a través del desarrollo de agencia personal y empoderamiento intrínseco. Universitas Psychologica, 10 (2), 327-340.

Chirinos, N. (2009). Características generacionales y los valores. Su impacto en lo laboral. Observatorio Laboral, 2(4). 133-153

Crasto, C., Marín-González, F. y Senior, A (2016). Responsabilidad Social en la gestión universitaria: una construcción colectiva. Espacio Abierto. 25(2), $185-208$

Díaz, R. (1979). La Psicología del Mexicano. México, D. F.: Trillas.

Duchscher, B. y Cowin, L. (2004). Multigenerational nurses in the workplace. JONA, 34(11). 493-501. Recuperado de https://www.ncbi.nlm.nih. gov/pubmed/15586070
Emakunde, V-G, (2013). La situación del proceso de empoderamiento de las mujeres "adultas" en el país Vasco. Vitoria-Gasteiz: Instituto Vasco de la Mujer.

Erazo, M., Jiménez M. y Lopéz, C. (2014). Empoderamiento y liderazgo femenino; su papel en la autogestión comunitaria en el corregimiento El Hormiguero - Valle del Cauca. Avances en Psicología Latinoamericana, 32(1).149-157. https://doi.org/dx.doi. org/10.12804/apl32.1.2014.10

Escudero, K., Serrano-Barquín, R., Segrado, R., Serrano, C. y López, E. (2017). Empoderamiento de la mujer en establecimientos de hospedaje en Metepec estado de México. Gran Tour, 15(1). 3-25. Recuperado de http://hdl. handle.net/20.500.11799/68060

Ferreiro, R. (2006). El reto de la educación del siglo XXI: La generación N. Apertura, 6 (5). 7285.

Furlow, N. (2011). Find us on Facebook: How Cause Marketing has Embraced Social Media. Journal of Marketing Development and Competitiveness, 5(6). 61-64.

Hernández, J. y García, R. (2015). Empoderamiento en mujeres mexicanas: Experiencias de mujeres líderes de México. De género: de la igualdad y la diferencia, 10. 419-434.

Hernández, J. y García, R. (2011). Mujeres mexicanas, empoderamiento $\mathrm{y}$ política. Revista electrónica de ciencias sociales, 3(10). 1886- 8452.

Hernández, J. y García, R. (2008). Instrumento para Medir el Empoderamiento de la Mujer. Tabasco: Universidad Juárez Autónoma de Tabasco.

Martin, C. (2005). From high maintenance to high productivity: What managers need to know about Generation Y. Industrial and Commercial Training, 37,(1) 39-44. 
Mayoux, L. (1997). The magic ingredient? Microfinance and women's empowerment. Washington DC: Campaña de la Cumbre del Microcrédito.

McGuire, D. Todnem By, R. y Hutchings, K. (2007). Towards a model of human resource solutions for achieving intergenerational interaction in organizations. Journal of European Industrial Training, 31, 592-608.

Ogg, J. \& Bonvalet, C. (2006). The babyboomer generation and the birth cohort of 1945- 1954: a European perspective. ESRG-AHRC. Available in https://youngfoundation.org/wpcontent/uploads/2013/06/03_06_Europeanbabyboomers.pdf

Pérez, M. y Vázquez, V. (2009). Familia y empoderamiento femenino: Ingresos, trabajo doméstico y libertad de movimiento de mujeres chontales de Nacajuca, Tabasco. Convergencia, 16(50), 187-218. Recuperado de http://repositoriodigital.academica. $\mathrm{mx} / \mathrm{jspui} / \mathrm{handle} / 987654321 / 476669$

Pick, S., García, R. y Leenen, I. (2011). Modelo para la promoción de la salud en comunidades rurales a través del desarrollo de agencia personal y empoderamiento intrínseco. Univ. Psychol, 10(2). 327-340.

Pick, S. \& Sirkin, J. (2010). Agentic Empowerment. New York: Osford University Press.

Pick, S., Sirkin, J., Ortega, I., Osorio, P., Martínez, R., Xocolotzin, U. y Givaudan, M. (2007). Escala Para Medir Agencia Personal y Empoderamiento (ESAGE). Interamerican Journal of Psychology, 41(3). 295-304.

Pincheira, A., y Arenas, T. (2016). Caracterización de los profesionales de la "generación millennials" de Arica y Parinacota, Chile, desde una mirada del capital intelectual. Interciencia, 41(12), 812-818.
Robles, A. (2015). Propuesta de convivencia escolar a través de talleres de prevención de violencia escolar con perspectiva de género. Cultura Educación y Sociedad, 6(2). 29-49.

Sánchez, A. (2006). El Feminismo en la construcción de la ciudadanía de las mujeres en México. Revista Itinerario de la Miradas, 63, 1-17. Recuperado de https://catedraunescodh. unam.mx//catedra/CONACYT/03. Curso_PGJ/Contenidos/contenidos/ Modulo_4/2_Alma\%20Rosa\%20Sanchez.pdf

Schuler, Mt. (1997). Los derechos de las mujeres son derechos humanos: La agenda internacional del empoderamiento. M. León, Poder y Empoderamiento de las Mujeres. Bogotá, D.C.: Tercer Mundo.

Smola, K. y Sutton, C. (2002). Generational differences: Revisiting generational work values for the new millennium. Journal of Organizational Behavior, 23(1). 363-382.

Vidal, F. (2015). La participación política en México: Entendiendo la desigualdad entre hombres y mujeres. Revista Mexicana de Ciencias Politicas y Sociales, 60(223), 317 355. Recuperado de http://www.redalyc.org/articulo.oa?id=42132948012 